\title{
Management of Intraventricular Hemorrhage
}

\author{
Holly E. Hinson, \\ Neurosciences Critical Care Division, The Johns Hopkins University School of Medicine, Meyer \\ 8-140, 600 North Wolfe Street, Baltimore, MD 21287, USA
}

Daniel F. Hanley, and

Neurosciences Critical Care Division, The Johns Hopkins University School of Medicine, Meyer 8-140, 600 North Wolfe Street, Baltimore, MD 21287, USA

\author{
Wendy C. Ziai \\ Neurosciences Critical Care Division, The Johns Hopkins University School of Medicine, Meyer \\ 8-140, 600 North Wolfe Street, Baltimore, MD 21287, USA
}

Holly E. Hinson: hhinson1@jhmi.edu; Daniel F. Hanley: dhanley@jhmi.edu; Wendy C. Ziai: weziai@jhmi.edu

\begin{abstract}
Brain hemorrhage is the most fatal form of stroke and has the highest morbidity of any stroke subtype. Intraventricular extension of hemorrhage (IVH) is a particularly poor prognostic sign, with expected mortality between $50 \%$ and $80 \%$. IVH is a significant and independent contributor to morbidity and mortality, yet therapy directed at ameliorating intraventricular clot has been limited. Conventional therapy centers on managing hypertension and intracranial pressure while correcting coagulopathy and avoiding complications such as rebleeding and hydrocephalus. Surgical therapy alone has not changed the natural history of the disease significantly. However, fibrinolysis in combination with extraventricular drainage shows promise as a technique to reduce intraventricular clot volume and to manage the concomitant complications of IVH.
\end{abstract}

\section{Keywords}

Intraventricular Hemorrhage; Intracerebral hemorrhage; External ventricular drainage

\section{Introduction}

\section{Epidemiology}

Brain hemorrhage has the highest morbidity and mortality of any stroke subtype.

Respectively, intracerebral hemorrhage (ICH) and subarachnoid hemorrhage (SAH) account for about $15 \%$ and $5 \%$ of the 750,000 strokes occurring yearly in the United States, totaling more than 45,000 patients per year [1-3]. About $45 \%$ of spontaneous ICHs and $25 \%$ of aneurysmal SAHs extend into the ventricles $[1,4,5]$. For patients with both ICH and intraventricular hemorrhage (IVH), the expected mortality is $50 \%$ to $80 \%$ [6, 7]. Patients with IVH are twice as likely to have poor outcomes (a modified Rankin scale [mRS] score of 4-6 at hospital discharge) and nearly three times more likely to die than their cohorts without IVH [8].

\footnotetext{
(C) Springer Science+Business Media, LLC 2010

Correspondence to: Daniel F. Hanley, dhanley a jhmi . edu.

Disclosure No other potential conflicts of interest relevant to this article were reported.
} 


\section{Pathophysiology}

Primary IVH is confined to the ventricular system, arising from an intraventricular source or a lesion contiguous to the ventricles. Examples include intraventricular trauma, aneurysm, vascular malformation, and tumor, usually involving the choroid plexus. Approximately $70 \%$ of IVHs are secondary; secondary IVHs may occur as an extension of an intraparenchymal hemorrhage or SAH into the ventricular system. Risk factors for IVH include older age, higher baseline ICH volume, mean arterial pressure values greater than $120 \mathrm{~mm} \mathrm{Hg}$, and location of the primary ICH [7]. Deep, subcortical structures tend to be most at risk for IVH; frequent locations include the putamen (35\%-50\%), lobes (30\%), thalamus $(10 \%-15 \%)$, pons $(5 \%-12 \%)$, caudate $(7 \%)$, and cerebellum $(5 \%)$ [8]. Whereas some authors have focused on the volume of the original ICH as the predictor of poor outcome, others have used sophisticated volumetrics to define a threshold volume of IVH $(20 \mathrm{~mL})$ as particularly ominous [9]. Hallevi et al. [10••] correlated larger ICH volume with the presence of IVH, as well as location near the ventricular system, which likely leads to early intraventricular rupture.

\section{Scoring Systems}

Several scoring systems were developed to calculate the amount and hence the severity of IVH. Although software-dependent volumetric analysis remains the gold standard, it is impractical for clinical bedside use. Several scales have gained some traction either in the literature (Graeb scale) or through clinical trials (expanded Graeb scale). The Graeb score is calculated by grading the lateral ventricles separately $(1=$ trace blood, $2=$ less than half the ventricle filled with blood, $3=$ more than half the ventricle filled with blood, $4=$ ventricle filled with blood and expanded) and adding that sum to scores for the third and fourth ventricles, which also are summed separately $(1=$ blood present, ventricle normal size; $2=$ ventricle filled with blood and expanded) [11]. The maximum score is 12. The Graeb scale is the most commonly reported scale in adults and correlates significantly with short-term outcome (Glasgow Outcome Score at 1 month) [12]. One study found that among patients with an ICH volume greater than $60 \mathrm{~cm}^{3}$, a Graeb score $\geq 6$ was significantly associated with acute hydrocephalus whereas a score $\leq 5$ was associated with a Glasgow coma scale (GCS) score $\geq 12$ on admission [13]. The expanded Graeb score (Table 1) is a modification with estimated IVH volume divided into quartiles and extra points given for expansion of separate ventricle compartments. The maximum score is 32 . It is not currently validated. A more recent IVH grading system proposed by Hallevi et al. [10••] is similar to the Graeb score with respect to assigning a number to each lateral ventricle based on amount of blood ( 1 = up to one-third filling, $2=$ one-third to two-thirds filling, and $3=>$ two-thirds filling) and adds 1 point for the presence of hydrocephalus. The third and fourth ventricles each are assigned a score of 0 for no blood and 1 for partial or complete filling with blood. The IVH score (IVHS) ranges from 0 to 23 and can be converted to IVH volume using a logarithmic transformation [IVH volume $(\mathrm{mL})=\mathrm{e}^{\mathrm{IVHS} / 5}$ ]. The IVHS was validated retrospectively, and when it was added to ICH volume to produce total hemorrhage volume, it improved the predictive power for poor outcome and mortality over both the Graeb score and the ICH score by Hemphill et al. [14]. However, the IVHS still must be validated prospectively.

\section{Prognosis}

Tuhrim et al. $[15,16]$ initially confirmed IVH as an independent risk factor for 30-day mortality after ICH and developed a model in which IVH volume contributed significantly to outcome prediction together with GCS score. Patients with IVH in addition to ICH had a lower initial GCS score and larger ICH volume. Other contributors to outcome included the number of ventricles containing blood and blood in the fourth ventricle. Subsequent prospective studies have continued to define the presence of IVH as an independent risk factor for mortality and poor functional outcome $[14,17]$. The Tuhrim algorithm continues 
to perform better than any other outcome prediction algorithm when compared directly with independent data sets [18].

Recently, three large randomized controlled trials reconfirmed IVH as a severity factor. The Surgical Trial in ICH (STICH) enrolled 964 subjects to test the value of early surgery versus medical management. IVH occurred in $42 \%$ of STICH subjects ( $n=377$ ); IVH with hydrocephalus occurred in $23 \%(n=208)$. Both were strongly associated with poor outcome: $31 \%$ of patients without IVH and $15 \%$ with IVH had a good outcome $(P<0.00001)$ [19]. When IVH and hydrocephalus were combined, the percentage of patients with a good outcome fell to $11 \%$. Similarly, in the phase $2 \mathrm{~b}$ proof-of-concept study of recombinant activated factor VII (rFVIIa) for ICH $(n=399)$, IVH occurred in $49 \%$ of subjects, in whom mRS scores were consistently worse; $80 \%$ of these subjects were vegetative or dead [20]. In the multicenter Factor Seven for Acute Hemorrhagic Stroke (FAST) trial, the presence of IVH again was associated with increased mortality [21•].

Blood in the ventricular system contributes to morbidity in a variety of ways. Damage to the reticular activating system and thalamus during the acute phase of hemorrhage expansion causes a decreased level of consciousness. Coma appears to be prolonged with both a larger volume of blood in the ventricles and a longer exposure [22, 23]. Ventricular blood clots blocking cerebrospinal fluid (CSF) conduits cause acute obstructive hydrocephalus, an immediate life-threatening condition limiting cerebral perfusion and potentially contributing to mass effect and resultant cerebral edema. Blood degradation products become embedded in the arachnoid granulations and may cause permanent occlusion and scarring, inhibiting CSF absorption and causing nonobstructive (communicating) hydrocephalus. Clotted blood may persist in the ventricles for several reasons related to coagulation and fibrinolytic pathways in the ventricular system. After neonatal IVH, tissue plasminogen activator may be demonstrated [24], but fibrinolytic activity often is not seen for several weeks after IVH and there appear to be insufficient concentrations of CSF plasminogen acutely [25]. Rapid immediate evolution of blood clot resolution in the ventricles therefore may be impaired by the fibrinolytic state of the CSF. In the study by Naff et al. [26] of the kinetics of intraventricular clot resolution in 17 patients with IVH, the percentage rate of clot resolution over the first 10 days was $10.8 \%$ per day.

The inflammatory reaction caused by blood breakdown products in the ventricles also may affect long-term cognitive function independent of clot volume or mass effect, as demonstrated in experimental IVH [22, 27]. In human IVH, significant cognitive deficits in SAH patients with IVH compared with those without IVH have been found on neuropsychological testing [28].

\section{Conventional Therapy Emergency Care}

A depressed level of consciousness may lead to loss of neurologic reflexes protecting a patent airway, placing the patient at risk for hypoxia, hypercapnia, and aspiration [29]. If endotracheal intubation and mechanical ventilation are required, sedatives and neuromuscular blocking agents that do not elevate intracranial pressure (ICP) should be selected [30].

Although elevated blood pressure may be a protective mechanism (Cushing-Kocher response) to preserve cerebral perfusion, it also may increase the risk of ongoing bleeding from ruptured small arteries and arterioles. Overaggressive treatment of blood pressure may decrease cerebral perfusion pressure and promote focal ischemia adjacent to hematoma. There is little prospective evidence in ICH to support a specific blood pressure threshold for 
all patients. The 2007 American Heart Association/American Stroke Association guidelines recommend treating systolic blood pressure (SBP) greater than $180 \mathrm{~mm} \mathrm{Hg}$ or diastolic blood pressure greater than $105 \mathrm{~mm} \mathrm{Hg}$ [31•]. Target SBP goals $\geq 160 \mathrm{~mm} \mathrm{Hg}$ are associated with hematoma enlargement compared with SBP goals $\leq 150 \mathrm{~mm} \mathrm{Hg}$ [32]. Of note, poorly controlled diabetes and SBP greater than $200 \mathrm{~mm} \mathrm{Hg}$ at admission portend a high risk of hematoma expansion [33]. Finally, the phase 3 portion of the randomized international INTERACT (Intensive Blood Pressure Reduction in Acute Cerebral Hemorrhage) study is determining whether lowering high blood pressure levels after the start of ICH reduces mortality or survival with a long-term disability. The initial "run-in" phase of this trial compared early intensive SBP lowering (target $<140 \mathrm{~mm} \mathrm{Hg}$ ) with the standard guideline (target $<180 \mathrm{~mm} \mathrm{Hg}$ ). Mean proportional ICH growth at $24 \mathrm{~h}$ was $36.3 \%$ in the group receiving guideline treatment versus $13.7 \%$ in the intensive therapy group. The absolute difference in ICH volume between the groups was $1.7 \mathrm{~mL}$. The absolute risk reduction of hematoma growth was $8 \%$ in the intensive therapy group [34•]. There currently are no data on the impact of blood pressure control on the presence or size of IVH. Practitioners are encouraged to maintain cerebral perfusion pressure above $60 \mathrm{~mm} \mathrm{Hg}$ in the setting of hydrocephalus and increased ICP [31, 35•].

Coagulopathy is associated with ICH and should be corrected as rapidly as possible. Anticoagulation with warfarin raises the risk of intracranial hemorrhage 7- to 10-fold [36]. Treating to an international normalized ratio of 1.4 may reduce the risk of progressive bleeding and clinical deterioration. Time to treatment with reversal agents is an important determinant of 24-hour anticoagulation reversal. It is recommended that a fresh frozen plasma infusion followed by oral vitamin $\mathrm{K}$ be given without delay in the emergency department to manage warfarin-related ICH [37]. Alternately, prothrombin complex concentrate and intravenous vitamin $\mathrm{K}$ may be given if volume overload is a concern. Additionally, there is evidence that reduced platelet activity is associated with larger IVH volume, which may relate to aspirin use [38]. In a prospective cohort of 73 patients with spontaneous ICH, 36 of whom had IVH, higher Graeb scores (IVH severity) were associated with less platelet activity (VerifyNow-Aspirin assay; Accumetrics, San Diego, CA) after correcting for ICH volume and location. At present, there is no robust evidence for transfusing platelets in patients taking platelet inhibitors such as aspirin and clopidogrel.

\section{Intensive Care Unit Care}

Care continues in the intensive care unit (ICU) setting. Patients should have the benefit of conventional neurologic ICU care including resuscitation with intravenous fluids, placement of the head of bed at $30^{\circ}$, correction of fever with antipyretics, and deep venous thrombosis prophylaxis with sequential compression devices and/or compression stockings. Low-dose prophylactic anticoagulation should be initiated $48 \mathrm{~h}$ after injury [39].

\section{Seizure Prevention}

Patients with ICH, particularly lobar ICH, are at risk for seizure; their 30-day post-ICH risk is quoted at approximately $8 \%$ [40]. Clinical and electrographic seizures should be aggressively treated, but antiepileptic drug (AED) prophylaxis is more controversial. AEDs frequently are used prophylactically in ICH to avoid seizure-associated neurologic deterioration and rebleeding. The 2007 American Heart Association guidelines for ICH care advise that a "brief" period of AED prophylaxis may be administered to prevent early seizures in lobar ICH [31•]. However, emerging evidence suggests this might not be necessary in all cases. Analysis of the placebo arm of the randomized, placebo-controlled, neuroprotectant Cerebral Hemorrhage and NXY-059 Trial (CHANT) indicated that the early use of AEDs following acute ICH was strongly associated with severe disability and death, independent of other significant predictors of poor outcome from ICH. Thirty-four percent 
( $n=100)$ of the 291 patients in this study also had IVH, and half the group $(52 \%, n=43)$ had a poor outcome (mRs score, 5-6). The authors point out that most of the patients received phenytoin as the AED, and speculate that this agent may impair functional outcome in humans as it does in animals [41]. Thus, prophylactic AEDs should be chosen and administered judiciously to patients at the highest risk for seizure.

\section{Hematoma Expansion}

Hematoma growth is an independent determinant of both mortality and functional outcome after ICH [42]. In a secondary analysis of data from the multicenter, randomized, placebocontrolled trial of rFVIIa effectiveness in spontaneous ICH, 170 of 374 patients (45\%) had IVH at baseline and 12\% (44 of 374) had a greater than $2 \mathrm{~mL}$ increase in IVH volume between baseline and 24-hour CT scan [7]. Limiting intraventricular hematoma growth may be an important therapeutic target.

\section{Management of Intracranial Pressure}

Often it is assumed that coma and death associated with IVH result from an acute rise in ICP that injures the reticular activating system or compromises cerebral perfusion. Nevertheless, no correlation between ICP and outcome has been reported for IVH. Diringer et al. [43] found that hydrocephalus increased mortality and produced higher intubation rates in patients with IVH. A Japanese study of 35 patients with IVH and ICH found that IVH severity influenced the occurrence of acute hydrocephalus and initial level of consciousness, which was significantly associated with prognosis. The authors concluded that priority treatment of the IVH should be given to patients with a Graeb score $\geq 6$ [12]. Adams and Diringer [44] reported that control of initial ICP with an external ventricular drain (EVD) had little impact on ventricular size or level of consciousness in their cohort of 22 patients. The authors speculated that parenchymal and/or IVH clot volume may have overshadowed the influence of hydrocephalus. Mortality in this series was 73\%. Coplin et al. [6] reported on a cohort of 40 patients with spontaneous IVH who received EVDs. The mean initial ICP was $15.6 \mathrm{~mm} \mathrm{Hg}$, and only six patients (15\%) had ICP elevation (ICP > $20 \mathrm{~mm} \mathrm{Hg}$ ) at the time of EVD placement. We analyzed every-4-hour ICP readings from $11 \mathrm{IVH}$ patients who required an EVD and found that initial ICP at the time of EVD placement was uncommonly elevated (1 of 11 patients) despite acute obstructive hydrocephalus [45]. ICP elevation greater than $20 \mathrm{~mm} \mathrm{Hg}$ occurred in only 14\% of observations during EVD use, and the EVD was equally effective in controlling ICP in patients treated with intraventricular urokinase or placebo. In this study, only 2 of 141 intraventricular injections of study agent, followed by EVD closure for $1 \mathrm{~h}$, were not tolerated and required reopening of the EVD. All these results support the concept that acute obstructive hydrocephalus remains a potentially lethal complication of IVH but that ICP may not be the only factor determining the harmful effect of IVH. The presence of a ventricular drain, which is kept open to drain at a clinically rational height, may explain the low incidence of ICP greater than $20 \mathrm{~mm} \mathrm{Hg}$. However, in this study, patients were required to have an intraparenchymal hematoma volume less than $30 \mathrm{~mL}$ and no significant coagulopathy or aneurysmal or arteriovenous malformationrelated ICH. These latter populations frequently experience intracranial hypertension; therefore, the significance of ICP across the full spectrum of IVH patients is not known.

What guidelines can be drawn regarding indications for intraventricular catheter (IVC) use in the IVH population? Although IVC placement should not be delayed in patients with severe IVH and neurologic deterioration secondary to acute hydrocephalus, a systematic review of observational studies of IVH in either SAH or ICH found that poor outcome (mRS score, 4 or 5) occurred in $90 \%$ of patients with conservative management and $89 \%$ with ventricular drainage only (no fibrinolytic therapy), suggesting that ventricular drainage alone may not have a beneficial effect in patients with spontaneous ICH and associated IVH (Fig. 
1) [46]. EVD alone was associated with a lower case fatality rate compared with conservative treatment (58\% vs $78 \%$ ), which may reflect early withdrawal of care in the conservative group. From a practice standpoint, there are many issues surrounding the effective use of EVDs, including catheter position relative to the side of the most IVH, timing of catheter placement, maintenance of IVC patency, and whether an EVD is even placed. From the older literature, Tuhrim et al. [16] reported an IVC usage rate of $1.6 \%$ in 129 patients, 47 (36\%) of whom had some degree of IVH. More recently, Steiner et al. [7] reported IVC use in 7.6\% of 170 patients with IVH, and Mendelow et al. [19] reported IVC use in $7.5 \%$ of patients in the STICH trial. Although the proportion of patients with acute obstructive hydrocephalus in these studies is unknown, the apparently infrequent use of IVCs in these multicenter clinical trials suggests there likely is clinical equipoise as to their benefit.

Generally, IVC placement is well tolerated, with a relatively low incidence of serious complications. The most frequent complications are hemorrhage, (intraparenchymal, intraventricular, or subdural), catheter-related infection, and obstruction requiring reinsertion. Catheter-related hemorrhages reportedly occur in $1 \%$ to $33 \%$ of patients $[2,7$, $29,30,47-49]$. This wide range likely is a result of the lack of a standard definition or imaging technique. We propose defining catheter tract hemorrhages by size (small, $<5 \mathrm{~mm}$; large $>5 \mathrm{~mm}$ ) and whether they are symptomatic or asymptomatic. IVC-related infection has been reported in 1-12\% of patients [12, 36-39, 51]. Currently, antibiotics are administered prophylactically either perioperatively or continuously, although previous investigations have not demonstrated an advantage of one strategy over the other. Our practice is to give a single periprocedural dose of oxacillin. Malpositioning of IVCs is not infrequent but clearly depends on definition. In a study of 169 EVD and 43 shunt procedures at the Mayo Clinic, 26 ventriculostomy catheters (12.3\%) were malpositioned as determined by postprocedural imaging [49]. Most misplacements involved an intraparenchymal distal catheter tip, whereas five were in other extraventricular spaces. The need for IVC replacement is common after IVH, usually because of blood clots occluding the catheter. In a prospective case-control study of 59 patients with IVH requiring an IVC, the catheter had to be replaced in $59 \%$ of patients who did not receive intraventricular thrombolytic therapy and $32 \%$ of those who did [51].

Placing a second catheter has emerged as a strategy to overcome problems related to unfavorable initial IVC position relative to the greatest IVH volume. Approximately $80 \%$ of IVCs are placed contralateral to the lateral ventricle with greatest IVH volume to minimize the chance of obstruction. Although placing the IVC into freely circulating CSF may be the goal of the initial IVC, to control ICP, a single catheter may be ineffective in removing substantial hematoma from the contralateral side, especially if the foramina of Munro are obstructed. It has been proposed, but not validated, that dual catheters may be indicated for larger clots producing trapped ventricles, clinically important shift, or pan-ventricular enlargement with cortical effacement/ischemia. These indications seem more frequent in patients with an IVH larger than $40 \mathrm{~mL}$. In this setting, surgical placement options include image guidance with operating room instrumentation.

\section{Intraventricular Thrombolysis}

Although ventriculostomy appears to be effective in controlling ICP, this technique does little to reduce morbidity and does not address the inflammatory process. The severity of communicating hydrocephalus appears to be related to IVH volume and the duration of exposure of CSF to clotted blood [52-56]. To address the mechanical and biochemical factors that likely contribute to brain tissue injury, thrombolytic-mediated removal of clot emerged as a pragmatic solution to facilitate blood clot removal and thereby prevent hydrocephalus and inflammation (Fig. 2). 
In the experimental literature, canine and porcine IVH models showed that larger volumes of blood clot injected into the ventricles were associated with a higher likelihood of mortality and that prolonged exposure of the ventricles to blood caused altered consciousness and pathologic changes of subarachnoid fibrosis, extensive ependymal cell loss, and subependymal glial proliferation on the lateral ventricle walls, most likely because of an inflammatory response independent of mass effect or hydrocephalus. Pang et al. [22] used the canine model to show that thrombolysis of blood injected into the ventricles with urokinase hastened the resolution of the intraventricular clot, promoted rapid return of consciousness, and improved neurologic outcome. Mayfrank et al. [57] showed similar results in a pig model of IVH using recombinant tissue plasminogen activator (rt-PA) as the lytic agent. These models have shown the ability of thrombolytic therapy to reverse or prevent ventricular enlargement, herniation, and coma; white cell infiltrates; and periventricular and generalized edema [58-60]. These findings provide the underlying biological premise for translating animal models to clinical treatment of IVH.

The first use of intraventricular rt-PA in humans appeared in the literature in 1991 [61]. Beneficial effects on clot resolution, ICP, ventricular size, and mortality have been seen in observational studies with both rt-PA and urokinase, although urokinase is not currently available in the United States [6, 62-64]. At present, intraventricular rt-PA for severe IVH is not approved by the US Food and Drug Administration, and its use in this capacity is considered off-label.

From 1990 to 2005, there were 230 published cases of thrombolysis for IVH, several of which suggest thrombolytics are safe and feasible. However, none of these studies provides conclusive evidence about its efficacy. In the 1990s, urokinase was used in several preliminary trials, with notable success. A prospective randomized trial using urokinase, 25,000 IU every $12 \mathrm{~h}$ until clot resolution, demonstrated earlier clot resolution in the treated group. The estimated mean time to achieve a clot $50 \%$ of its original size was 4.7 days for the urokinase group and 8.5 days for the placebo group [65]. Other case series with urokinase also demonstrated improved outcomes compared with historical controls [6, 66]. However, urokinase was withdrawn from the market because of safety concerns, and rt-PA became the thrombolytic of choice for IVH. In 2002, Lapointe and Haines [67] reviewed the safety and efficacy of thrombolysis in the Cochrane collaboration format. The authors identified seven independent studies using intra-ventricular thrombolytic agents in 74 patients with ICH or SAH. Urokinase was used in 17 patients and rt-PA in 57 at a dosage of 4 to $20 \mathrm{mg} / \mathrm{d}$. Good neurologic outcome was described in 50 of 74 patients. Complications included bacterial meningitis $(n=5)$, increased hematoma volume $(n=1)$, and extradural hematomas $(n=2)$. Although anecdotal evidence supported safety and possible therapeutic value, there was insufficient evidence to support clinical efficacy, and the consensus supported the need for a large randomized prospective study of rt-PA in IVH.

The Clot Lysis Evaluating Accelerated Resolution of Intraventricular Hemorrhage (CLEAR$\mathrm{IVH}$ ) trial is a phase 2 randomized clinical trial using rt-PA to treat IVH in patients with an $\mathrm{ICH}$ volume less than $30 \mathrm{~mL}$ (using the $\mathrm{ABC} / 2$ method). The trial investigated several questions, namely whether the rapid dissolution of the intraventricular hematoma with fibrinolytic agents would have the following benefits: 1) reduction of ICP, 2) increased cerebral perfusion, 3) low chance of chronic IVH-induced hydrocephalus and need for shunting, 4) reduced risk of vasospasm, and 5) decreased mortality rate and morbidity as a result of more rapid clearance of IVH and shortened duration of IVC drainage. Based on a dose- and dose interval-finding design, 100 patients (placebo, $n=22$; treatment, $n=78$ ) were given doses ranging from 0.3 to $3 \mathrm{mg}$ every 8 or $12 \mathrm{~h}$. Rates of ventriculitis, cerebritis, rebleeding, and 30-day mortality all were well below predetermined safety thresholds. In treated patients, it appears that rt-PA facilitates opening of the lower ventricular system, and 
once the clot is removed, clot lysis proceeds more rapidly compared with placebo. CLEAR III, a clinically determinant trial, began only recently, but preliminary analyses show that low doses of rt-PA $(1.0 \mathrm{mg})$ can be administered safely to patients with stable IVH clots and may increase lysis rates $[68 \cdot]$.

Intraventricular administration of rt-PA requires sterile technique and preparation in preservative-free solution. Once lyophilized drug in the vial is reconstituted, it must be used within $8 \mathrm{~h}$, although the preparation may be frozen for up to $24 \mathrm{~h}$. Among reported case series, the rt-PA dose ranges from 0.3- to 8-mg boluses, with total cumulative doses up to 32 $\mathrm{mg}$. The dosing frequency ranges from once daily to every $6 \mathrm{~h}$. Pharmacokinetic data suggest that the half-life of rt-PA in the ventricular cavity is approximately $3 \mathrm{~h}$. Most investigators have taken the practical approach of closing the ventricular drainage system for $1 \mathrm{~h}$ after fibrinolytic instillation to allow for drug-clot interactions and to optimize clot lysis. The influence of catheter placement and appropriate dosing on achieving optimal clot lysis will be revealed by the results from the CLEAR-IVH trial.

Several small studies of intraventricular or intracisternal thrombolysis in SAH patients with IVH have shown promising results. These studies seem to suggest that thrombolytic treatment hastens clearance of aneurysmal IVH, normalizes ICP, reduces IVC obstruction [69], and may prevent vasospasm. In several reported cases, intra-ventricular rt-PA was used safely after endovascular aneurysmal coiling [70]. Whether timely clearance of severe IVH with thrombolytics in high-grade SAH improves outcome and reduces the risk of vasospasm-related infarction is an area of interest for future research.

\section{Surgical Evacuation}

Surgical evacuation of parenchymal hematomas remains a controversial therapy. Of 902 patients followed up in the STICH trial, favorable outcomes were reported in $31 \%$ of those without IVH and $15 \%$ with IVH $(P<0.00001)$, regardless of hydrocephalus. Additionally, favorable outcomes were less frequent in patients with IVH and hydrocephalus $(12 \%)$ than in those with IVH alone (20\%, $P=0.031$ ). In patients with IVH, early surgical intervention resulted in more favorable outcomes (18\%) compared with initial conservative management (12\%), but this difference was not statistically significant $(P=0.141)$ [19]. In a post hoc analysis of 221 subjects with lobar ICH and no IVH, surgical intervention to remove the ICH resulted in an absolute 12\% improvement in functional outcome compared with medical treatment [71]. As in the FAST trial, there was no standard plan to remove IVH, and the low use of IVCs in this study may have masked the benefits of clot stabilization [72••].

As for ICH, various surgical procedures potentially are available for removing IVH. Although the conventional treatment is ventricular drainage, open craniotomy, and surgical evacuation of IVH, stereotactic drainage of IVH and minimally invasive treatments have limited experience in the literature. Zhang et al. [73] described their experience with 22 patients with IVH and less than $30 \mathrm{~mL}$ ICH who underwent neuroendoscopic aspiration of IVH within $48 \mathrm{~h}$ of onset. Patients with more than $10 \mathrm{~mL}$ of remaining IVH volume also received intraventricular urokinase. Cases were compared with a control group of 20 patients with IVH treated with EVD and intraventricular urokinase. In the neuroendoscopy group, removal of more than $90 \%$ of IVH or ICH was achieved on 24-hour CT scan in 15 of 22 cases, compared with less than $60 \%$ removal in three patients treated with EVD and almost no removal in the remaining 17 patients. Two patients in each group died of nonneurologic sequelae, and 2-month follow-up was good or excellent in 14 surgical patients compared with six control patients. Yadav et al. [74] described endoscopic evacuation of IVH in 25 patients with thalamic and putaminal ICH. Although resolution of hydrocephalus occurred in $96 \%$ of the patients, outcome depended predominantly on preoper-ative GCS score, with $81 \%$ having good recovery with a GCS score $\geq 9$ and $56 \%$ mortality if the initial 
GCS score was $\leq 8$. There are anecdotal reports and small series of patients undergoing open surgical evacuation of IVH. These include a case report of a patient with SAH and lifethreatening fourth ventricle dilation who underwent posterior fossa decompressive craniectomy and direct intraventricular clot evacuation, with a good neurologic result [75]. In a study of 74 patients with poor-grade SAH, 18 patients with "packed IVH" underwent evacuation of IVH via frontal corticotomy and postoperative irrigation of the ventricular drainage system with urokinase [76]. None of these patients had a favorable outcome, and seven died, which may explain why open procedures have not gained significant traction.

\section{Conclusions}

IVH may be a devastating complication associated with ICH, predicting worsened morbidity and mortality. Although conventional therapy has not made a dramatic impact on the natural history of the disease, several investigational techniques show promise in reducing the impact of IVH on recovery from ICH.

\section{Acknowledgments}

Dr. Hanley and Dr. Ziai are investigators in the CLEAR-IVH trial, which is sponsored by the US Food and Drug Administration's orphan drug program and assisted by a donation from Cathflo and a sponsored research agreement from Genentech (use patent: Johns Hopkins medical institutions).

\section{References}

Papers of particular interest, published recently, have been highlighted as:

- Of importance

•• Of major importance

1. Brott $\mathrm{T}$, Thalinger $\mathrm{K}$, Hertzberg V. Hypertension as a risk factor for spontaneous intracerebral hemorrhage. Stroke. 1986; 17 (6):1078-1083. [PubMed: 3810704]

2. Broderick JP, Brott T, Tomsick T, et al. Intracerebral hemorrhage more than twice as common as subarachnoid hemorrhage. J Neurosurg. 1993; 78(2):188-191. [PubMed: 8421201]

3. Woo D, Haverbusch M, Sekar P, et al. Effect of untreated hypertension on hemorrhagic stroke. Stroke. 2004; 35(7):1703-1708. [PubMed: 15155969]

4. Daverat P, Castel JP, Dartigues JF, Orgogozo JM. Death and functional outcome after spontaneous intracerebral hemorrhage. A prospective study of 166 cases using multivariate analysis. Stroke. 1991; 22(1):1-6. [PubMed: 1987664]

5. Mohr G, Ferguson G, Khan M, et al. Intraventricular hemorrhage from ruptured aneurysm. Retrospective analysis of 91 cases. J Neurosurg. 1983; 58(4):482-487. [PubMed: 6827343]

6. Coplin WM, Vinas FC, Agris JM, et al. A cohort study of the safety and feasibility of intraventricular urokinase for nonaneurysmal spontaneous intraventricular hemorrhage. Stroke. 1998; 29 (8):1573-1579. [PubMed: 9707195]

7. Steiner T, Diringer MN, Schneider D, et al. Dynamics of intraventricular hemorrhage in patients with spontaneous intracerebral hemorrhage: risk factors, clinical impact, and effect of hemostatic therapy with recombinant activated factor VII. Neurosurgery. 2006; 59(4):767-773. discussion 773-764. [PubMed: 17038942]

8. Hallevi H, Albright KC, Aronowski J, et al. Intraventricular hemorrhage: anatomic relationships and clinical implications. Neurology. 2008; 70(11):848-852. [PubMed: 18332342]

9. Young WB, Lee KP, Pessin MS, et al. Prognostic significance of ventricular blood in supratentorial hemorrhage: a volumetric study. Neurology. 1990; 40(4):616-619. [PubMed: 2320234]

$10 \bullet$. Hallevi H, Dar NS, Barreto AD, et al. The IVH score: a novel tool for estimating intraventricular hemorrhage volume: clinical and research implications. Crit Care Med. 2009; 37(3):969-974. 
e961. Hallevi and his group describe the relationship between ICH volume and anatomic proximity to IVH. [PubMed: 19237905]

11. Graeb DA, Robertson WD, Lapointe JS, et al. Computed tomographic diagnosis of intraventricular hemorrhage. Etiology and prognosis. Radiology. 1982; 143(1):91-96. [PubMed: 6977795]

12. Ruscalleda J, Peiro A. Prognostic factors in intraparenchymatous hematoma with ventricular hemorrhage. Neuroradiology. 1986; 28 (1):34-37. [PubMed: 3951686]

13. Nishikawa T, Ueba T, Kajiwara M, et al. A priority treatment of the intraventricular hemorrhage (IVH) should be performed in the patients suffering intracerebral hemorrhage with large IVH. Clin Neurol Neurosurg. 2009; 111(5):450-453. [PubMed: 19231066]

14. Hemphill JC 3rd, Bonovich DC, Besmertis L, et al. The ICH score: a simple, reliable grading scale for intracerebral hemorrhage. Stroke. 2001; 32(4):891-897. [PubMed: 11283388]

15. Tuhrim S, Dambrosia JM, Price TR, et al. Intracerebral hemorrhage: external validation and extension of a model for prediction of 30-day survival. Ann Neurol. 1991; 29(6):658-663. [PubMed: 1842899]

16. Tuhrim S, Horowitz DR, Sacher M, Godbold JH. Volume of ventricular blood is an important determinant of outcome in supratentorial intracerebral hemorrhage. Crit Care Med. 1999; 27 (3): 617-621. [PubMed: 10199544]

17. Mayfrank L, Hutter BO, Kohorst Y, et al. Influence of intraventricular hemorrhage on outcome after rupture of intracranial aneurysm. Neurosurg Rev. 2001; 24(4):185-191. [PubMed: 11778824]

18. Ariesen MJ, Algra A, van der Worp HB, Rinkel GJ. Applicability and relevance of models that predict short term outcome after intracerebral haemorrhage. J Neurol Neurosurg Psychiatry. 2005; 76(6):839-844. [PubMed: 15897508]

19. Mendelow AD, Gregson BA, Fernandes HM, et al. Early surgery versus initial conservative treatment in patients with spontaneous supratentorial intracerebral haematomas in the International Surgical Trial in Intracerebral Haemorrhage (STICH): a randomised trial. Lancet. 2005; 365(9457):387-397. [PubMed: 15680453]

20. Mayer SA, Brun NC, Begtrup K, et al. Recombinant activated factor VII for acute intracerebral hemorrhage. N Engl J Med. 2005; 352(8):777-785. [PubMed: 15728810]

21 . Mayer SA, Brun NC, Begtrup K, et al. Efficacy and safety of recombinant activated factor VII for acute intracerebral hemorrhage. N Engl J Med. 2008; 358(20):2127-2137. The FAST trial investigated hemostatic therapy with rFVIIa for ICH, which reduced hematoma growth but did not improve survival or functional outcome. Hematoma growth has important implications for both ICH and IVH. [PubMed: 18480205]

22. Pang D, Sclabassi RJ, Horton JA. Lysis of intraventricular blood clot with urokinase in a canine model: part 1. Canine intraventricular blood cast model. Neurosurgery. 1986; 19(4):540-546. [PubMed: 3491338]

23. Steinke W, Sacco RL, Mohr JP, et al. Thalamic stroke. Presentation and prognosis of infarcts and hemorrhages. Arch Neurol. 1992; 49(7):703-710. [PubMed: 1497496]

24. Whitelaw A, Mowinckel MC, Fellman V, Abildgaard U. Endogenous tissue plasminogen activator in neonatal cerebrospinal fluid. Eur J Pediatr. 1996; 155(2):117-119. [PubMed: 8775226]

25. Whitelaw A. Endogenous fibrinolysis in neonatal cerebrospinal fluid. Eur J Pediatr. 1993; 152(11): 928-930. [PubMed: 8276026]

26. Naff NJ, Williams MA, Rigamonti D, et al. Blood clot resolution in human cerebrospinal fluid: evidence of first-order kinetics. Neurosurgery. 2001; 49(3):614-619. discussion 619-621. [PubMed: 11523671]

27. Pang D, Sclabassi RJ, Horton JA. Lysis of intraventricular blood clot with urokinase in a canine model: part 2. In vivo safety study of intraventricular urokinase. Neurosurgery. 1986; 19(4):547552. [PubMed: 3491339]

28. Hutter BO, Kreitschmann-Andermahr I, Gilsbach JM. Cognitive deficits in the acute stage after subarachnoid hemorrhage. Neurosurgery. 1998; 43(5):1054-1065. [PubMed: 9802849]

29. Gujjar AR, Deibert E, Manno EM, et al. Mechanical ventilation for ischemic stroke and intracerebral hemorrhage: indications, timing, and outcome. Neurology. 1998; 51(2):447-451. [PubMed: 9710017] 
30. Roppolo LP, Walters K. Airway management in neurological emergencies. Neurocrit Care. 2004; 1(4):405-414. [PubMed: 16174942]

31•. Broderick J, Connolly S, Feldmann E, et al. Guidelines for the management of spontaneous intracerebral hemorrhage in adults: 2007 update: a guideline from the American Heart Association/American Stroke Association Stroke Council, High Blood Pressure Research Council, and the Quality of Care and Outcomes in Research Interdisciplinary Working Group. Circulation. 2007; 116(16):e391-e413. These guidelines for managing ICH form the standard of care for both ICH and IVH. [PubMed: 17938297]

32. Ohwaki K, Yano E, Nagashima H, et al. Blood pressure management in acute intracerebral hemorrhage: relationship between elevated blood pressure and hematoma enlargement. Stroke. 2004; 35(6):1364-1367. [PubMed: 15118169]

33. Kazui S, Naritomi H, Yamamoto H, et al. Enlargement of spontaneous intracerebral hemorrhage. Incidence and time course. Stroke. 1996; 27(10):1783-1787. [PubMed: 8841330]

34. Anderson CS, Huang Y, Wang JG, et al. Intensive blood pressure reduction in acute cerebral haemorrhage trial (INTERACT): a randomised pilot trial. Lancet Neurol. 2008; 7(5):391-399. The INTERACT study investigated the relationship among blood pressure, ICH, and hematoma growth. Limiting hematoma growth is an important goal in IVH as well as ICH. [PubMed: 18396107]

35•. Steiner T, Kaste M, Forsting M, et al. Recommendations for the management of intracranial haemorrhage_-part I: spontaneous intracerebral haemorrhage. The European Stroke Initiative Writing Committee and the Writing Committee for the EUSI Executive Committee. Cerebrovasc Dis. 2006; 22(4):294-316. The European Stroke Initiative guidelines are the European standards for treating ICH and IVH. [PubMed: 16926557]

36. Hart RG, Boop BS, Anderson DC. Oral anticoagulants and intracranial hemorrhage. Facts and hypotheses. Stroke. 1995; 26 (8):1471-1477. [PubMed: 7631356]

37. Goldstein JN, Thomas SH, Frontiero V, et al. Timing of fresh frozen plasma administration and rapid correction of coagulopathy in warfarin-related intracerebral hemorrhage. Stroke. 2006; 37 (1):151-155. [PubMed: 16306465]

38. Naidech AM, Bendok BR, Garg RK, et al. Reduced platelet activity is associated with more intraventricular hemorrhage. Neurosurgery. 2009; 65(4):684-688. discussion 688. [PubMed: 19834372]

39. Orken DN, Kenangil G, Ozkurt H, et al. Prevention of deep venous thrombosis and pulmonary embolism in patients with acute intracerebral hemorrhage. Neurologist. 2009; 15(6):329-331. [PubMed: 19901711]

40. Passero S, Rocchi R, Rossi S, et al. Seizures after spontaneous supratentorial intracerebral hemorrhage. Epilepsia. 2002; 43 (10):1175-1180. [PubMed: 12366733]

41. Messe SR, Sansing LH, Cucchiara BL, et al. Prophylactic antiepileptic drug use is associated with poor outcome following ICH. Neurocrit Care. 2009; 11(1):38-44. [PubMed: 19319701]

42. Davis SM, Broderick J, Hennerici M, et al. Hematoma growth is a determinant of mortality and poor outcome after intracerebral hemorrhage. Neurology. 2006; 66(8):1175-1181. [PubMed: 16636233]

43. Diringer MN, Edwards DF, Zazulia AR. Hydrocephalus: a previously unrecognized predictor of poor outcome from supratentorial intracerebral hemorrhage. Stroke. 1998; 29(7):1352-1357. [PubMed: 9660386]

44. Adams RE, Diringer MN. Response to external ventricular drainage in spontaneous intracerebral hemorrhage with hydrocephalus. Neurology. 1998; 50(2):519-523. [PubMed: 9484388]

45. Ziai WC, Torbey MT, Naff NJ, et al. Frequency of sustained intracranial pressure elevation during treatment of severe intraventricular hemorrhage. Cerebrovasc Dis. 2009; 27(4):403-410. [PubMed: 19295201]

46. Nieuwkamp DJ, de Gans K, Rinkel GJ, Algra A. Treatment and outcome of severe intraventricular extension in patients with subarachnoid or intracerebral hemorrhage: a systematic review of the literature. J Neurol. 2000; 247(2):117-121. [PubMed: 10751114] 
47. Tuhrim S, Horowitz DR, Sacher M, Godbold JH. Validation and comparison of models predicting survival following intracerebral hemorrhage. Crit Care Med. 1995; 23(5):950-954. [PubMed: 7736756]

48. LeRoux PD, Haglund MM, Newell DW, et al. Intraventricular hemorrhage in blunt head trauma: an analysis of 43 cases. Neurosurgery. 1992; 31(4):678-684. discussion 684-675. [PubMed: 1407453]

49. Saladino A, White JB, Wijdicks EF, Lanzino G. Malplacement of ventricular catheters by neurosurgeons: a single institution experience. Neurocrit Care. 2009; 10(2):248-252. [PubMed: 18923816]

50. Lozier AP, Sciacca RR, Romagnoli MF, Connolly ES Jr. Ventriculostomy-related infections: a critical review of the literature. Neurosurgery. 2002; 51(1):170-181. discussion 181-172. [PubMed: 12182415]

51. Huttner HB, Staykov D, Bardutzky J, et al. Treatment of intraventricular hemorrhage and hydrocephalus [in German]. Nervenarzt. 2008; 79(12):1369-1370. 1372-1364, 1376. [PubMed: 18626618]

52. Ahmann PA, Lazzara A, Dykes FD, et al. Intraventricular hemorrhage in the high-risk preterm infant: incidence and outcome. Ann Neurol. 1980; 7(2):118-124. [PubMed: 7369717]

53. Findlay JM, Weir BK, Gordon $P$, et al. Safety and efficacy of intrathecal thrombolytic therapy in a primate model of cerebral vasospasm. Neurosurgery. 1989; 24(4):491-498. [PubMed: 2496328]

54. Kassell NF, Torner JC, Adams HP Jr. Antifibrinolytic therapy in the acute period following aneurysmal subarachnoid hemorrhage. Preliminary observations from the Cooperative Aneurysm Study. J Neurosurg. 1984; 61(2):225-230. [PubMed: 6737046]

55. Papile LA, Burstein J, Burstein R, Koffler H. Incidence and evolution of subependymal and intraventricular hemorrhage: a study of infants with birth weights less than 1,500 gm. J Pediatr. 1978; 92(4):529-534. [PubMed: 305471]

56. Yasargil MG, Yonekawa Y, Zumstein B, Stahl HJ. Hydrocephalus following spontaneous subarachnoid hemorrhage. Clinical features and treatment. J Neurosurg. 1973; 39(4):474-479. [PubMed: 4730336]

57. Mayfrank L, Kissler J, Raoofi R, et al. Ventricular dilatation in experimental intraventricular hemorrhage in pigs. Characterization of cerebrospinal fluid dynamics and the effects of fibrinolytic treatment. Stroke. 1997; 28(1):141-148. [PubMed: 8996503]

58. Wagner KR, Xi G, Hua Y, et al. Ultra-early clot aspiration after lysis with tissue plasminogen activator in a porcine model of intracerebral hemorrhage: edema reduction and blood-brain barrier protection. J Neurosurg. 1999; 90(3):491-498. [PubMed: 10067918]

59. Mayfrank L, Kim Y, Kissler J, et al. Morphological changes following experimental intraventricular haemorrhage and intra-ventricular fibrinolytic treatment with recombinant tissue plasminogen activator. Acta Neuropathol. 2000; 100(5):561-567. [PubMed: 11045679]

60. Narayan RK, Narayan TM, Katz DA, et al. Lysis of intracranial hematomas with urokinase in a rabbit model. J Neurosurg. 1985; 62(4):580-586. [PubMed: 3973729]

61. Findlay JM, Weir BK, Stollery DE. Lysis of intraventricular hematoma with tissue plasminogen activator. Case report. J Neurosurg. 1991; 74(5):803-807. [PubMed: 1901601]

62. Findlay JM, Grace MG, Weir BK. Treatment of intraventricular hemorrhage with tissue plasminogen activator. Neurosurgery. 1993; 32(6):941-947. discussion 947. [PubMed: 8327096]

63. Andrews CO, Engelhard HH. Fibrinolytic therapy in intraventricular hemorrhage. Ann Pharmacother. 2001; 35(11):1435-1448. [PubMed: 11724097]

64. Naff NJ, Carhuapoma JR, Williams MA, et al. Treatment of intraventricular hemorrhage with urokinase: effects on 30-Day survival. Stroke. 2000; 31(4):841-847. [PubMed: 10753985]

65. Naff NJ, Hanley DF, Keyl PM, et al. Intraventricular thrombolysis speeds blood clot resolution: results of a pilot, prospective, randomized, double-blind, controlled trial. Neurosurgery. 2004; 54(3):577-583. discussion 583-574. [PubMed: 15028130]

66. Todo T, Usui M, Takakura K. Treatment of severe intraventricular hemorrhage by intraventricular infusion of urokinase. J Neurosurg. 1991; 74(1):81-86. [PubMed: 1984512]

67. Lapointe M, Haines S. Fibrinolytic therapy for intraventricular hemorrhage in adults. Cochrane Database Syst Rev. 2002; (3):CD003692. [PubMed: 12137707] 
68 . Morgan T, Awad I, Keyl P, et al. Preliminary report of the clot lysis evaluating accelerated resolution of intraventricular hemorrhage (CLEAR-IVH) clinical trial. Acta Neurochir Suppl. 2008; 105:217-220. This article presents preliminary data from the CLEAR-IVH trial. [PubMed: 19066112]

69. Findlay JM, Jacka MJ. Cohort study of intraventricular thrombolysis with recombinant tissue plasminogen activator for aneurysmal intraventricular hemorrhage. Neurosurgery. 2004; 55(3): 532-537. discussion 537-538. [PubMed: 15335420]

70. Hall B, Parker D Jr, Carhuapoma JR. Thrombolysis for intraventricular hemorrhage after endovascular aneurysmal coiling. Neurocrit Care. 2005; 3(2):153-156. [PubMed: 16174886]

71. Mendelow AD, Teasdale GM, Barer D, et al. Outcome assignment in the International Surgical Trial of Intracerebral Haemorrhage. Acta Neurochir (Wien). 2003; 145(8):679-681. discussion 681. [PubMed: 14520548]

72••. Hanley DF. Intraventricular hemorrhage: severity factor and treatment target in spontaneous intracerebral hemorrhage. Stroke. 2009; 40(4):1533-1538. This review article from our group discusses emerging IVH management principles. [PubMed: 19246695]

73. Zhang Z, Li X, Liu Y, et al. Application of neuroendoscopy in the treatment of intraventricular hemorrhage. Cerebrovasc Dis. 2007; 24(1):91-96. [PubMed: 17519550]

74. Yadav YR, Mukerji G, Shenoy R, et al. Endoscopic management of hypertensive intraventricular haemorrhage with obstructive hydrocephalus. BMC Neurol. 2007; 7:1. [PubMed: 17204141]

75. Lagares A, Putman CM, Ogilvy CS. Posterior fossa decompression and clot evacuation for fourth ventricle hemorrhage after aneurysmal rupture: case report. Neurosurgery. 2001; 49(1):208-211. [PubMed: 11440445]

76. Shimoda M, Oda S, Shibata M, et al. Results of early surgical evacuation of packed intraventricular hemorrhage from aneurysm rupture in patients with poor-grade subarachnoid hemorrhage. J Neurosurg. 1999; 91(3):408-414. [PubMed: 10470815] 


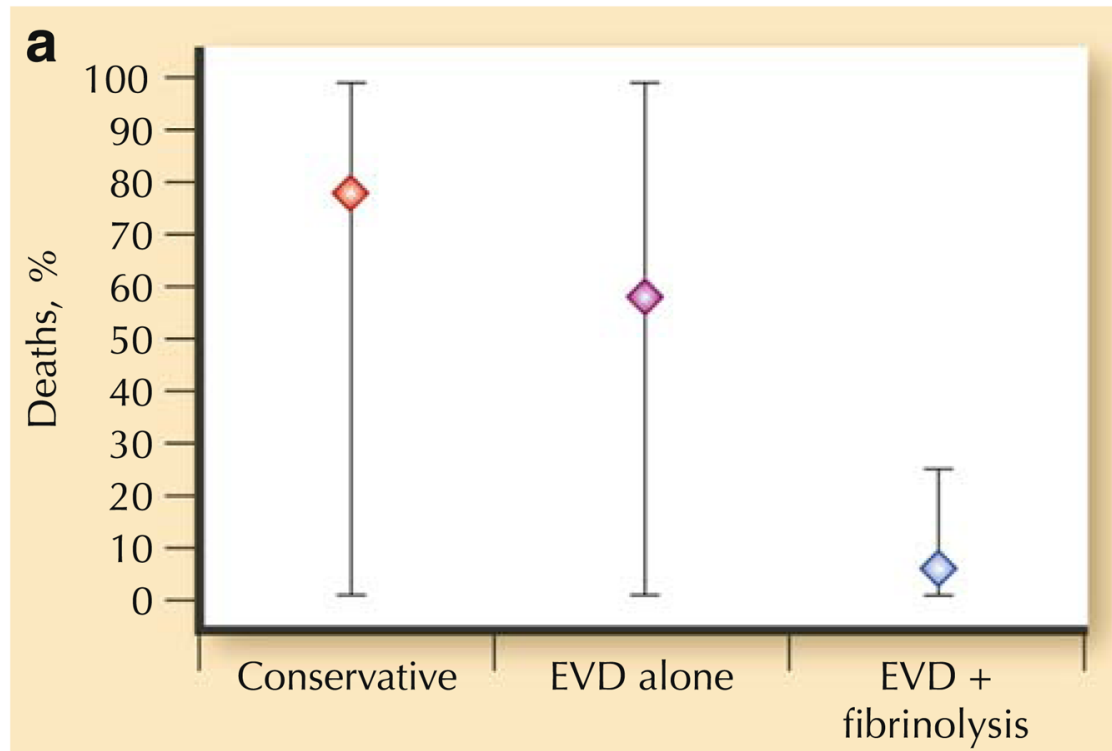

b

Treatment

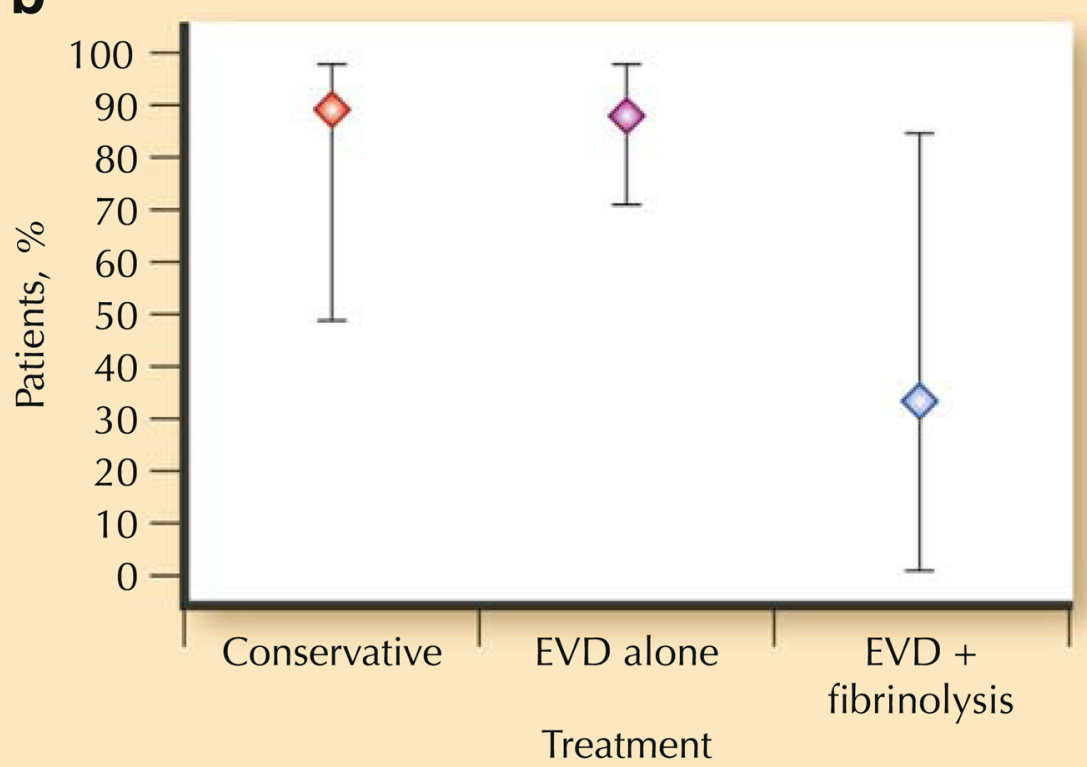

Fig. 1.

Severe intraventricular extension in patients with subarachnoid or intracerebral hemorrhage treated with conservative therapy, external ventricular drainage (EVD), and EVD with fibrinolysis, based on a systematic review of the literature. a Mortality. b Percentage of patients experiencing a poor outcome. (Adapted from Nieuwkamp et al. [46]) 


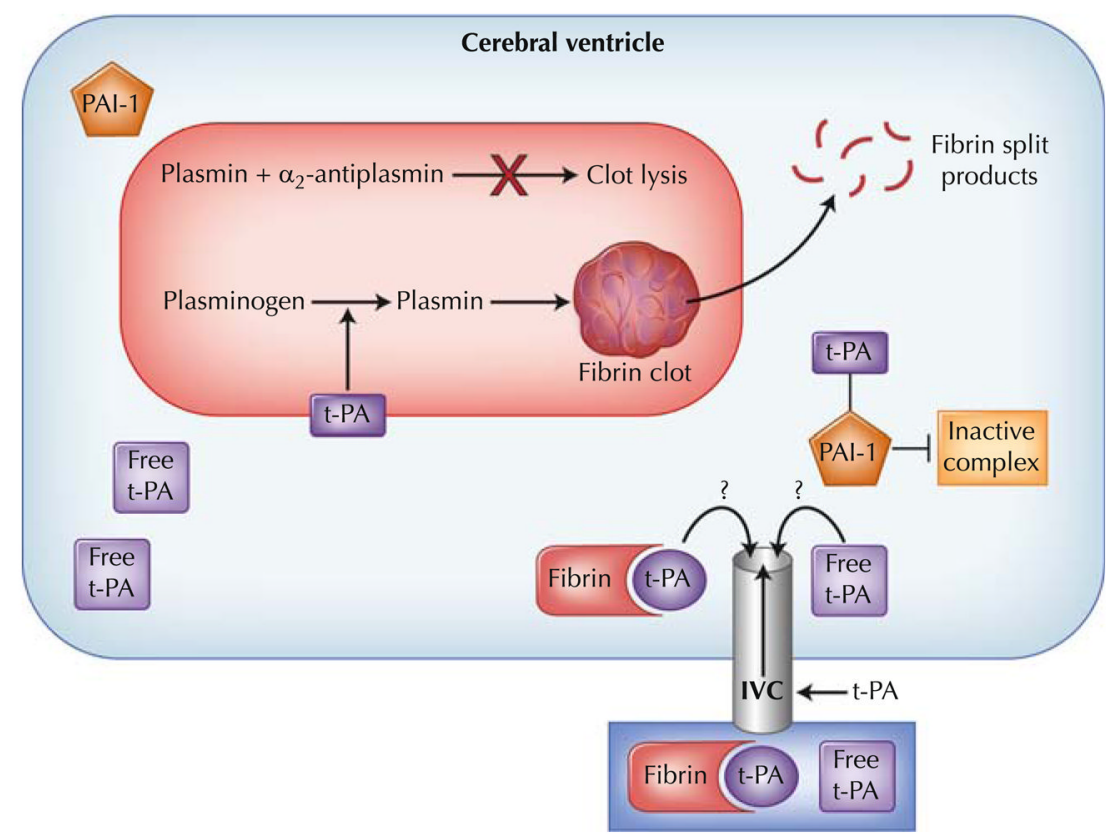

Fig. 2.

Biochemical reactions within the cerebral ventricle after administration of intraventricular tissue plasminogen activator (t-PA, alteplase) in patients with intraventricular hemorrhage (IVH). Fibrin in the blood clot provides binding sites for alteplase and plasminogen. On the fibrin surface, alteplase and intrinsic t-PA have a high affinity for plasminogen, which is converted to plasmin. Plasmin breaks down the blood clot with release of fibrin degradation products. Urokinase does not have specific affinity for fibrin and activates both freely circulating and fibrin-bound plasminogen. Fibrinolysis is terminated by circulating inhibitors of plasminogen, plasminogen activator inhibitor (PAI) 1 and 2; by inactivation of plasmin by $\alpha_{2}$-antiplasmin; and by clearance of fibrinolytic factors through the liver.

Normally, cerebrospinal fluid concentrations of plasminogen and t-PA are minute because of their high molecular weight. Plasminogen and t-PA incorporated within the clot itself likely are most responsible for clot resolution in IVH. IVC-intraventricular catheter 


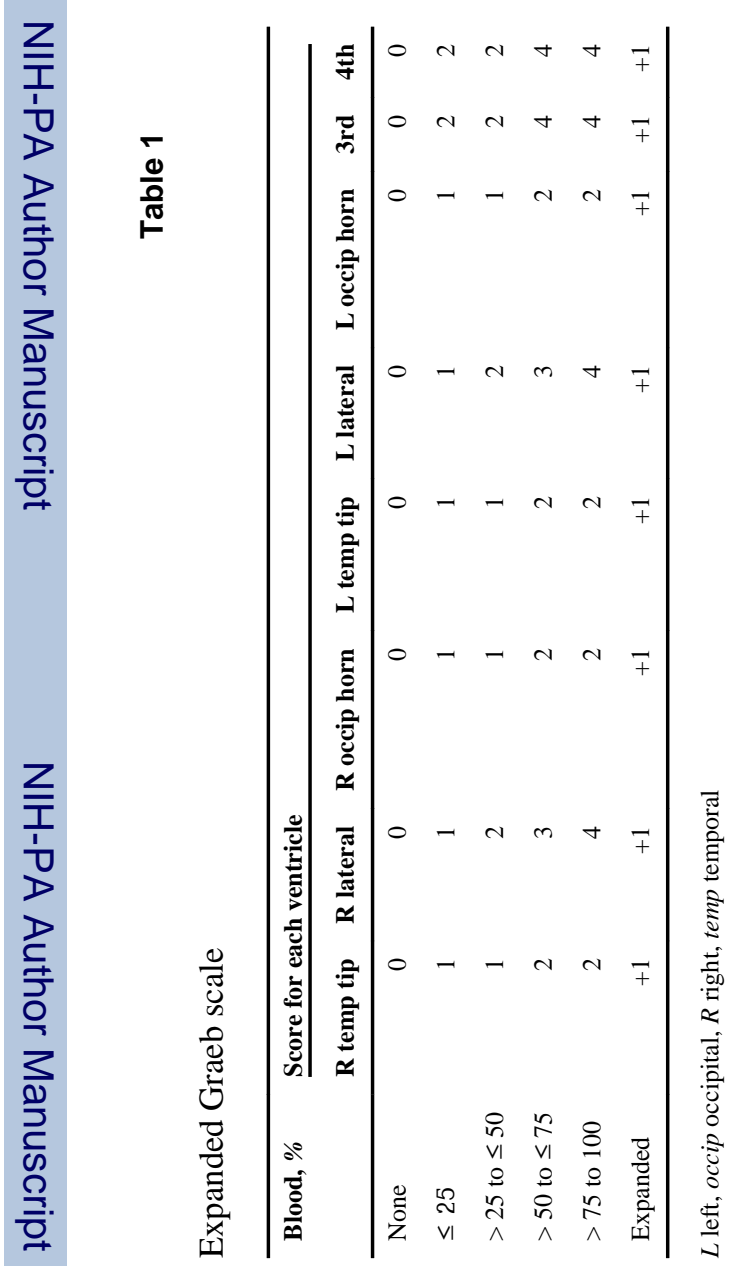

Curr Neurol Neurosci Rep. Author manuscript; available in PMC 2011 July 18. 